\title{
Smartphone Sensor-Based Activity Recognition by Using Machine Learning and Deep Learning Algorithms
}

\author{
Qingzhong Liu, Zhaoxian Zhou, Sarbagya Ratna Shakya, Prathyusha Uduthalapally, Mengyu Qiao, and \\ Andrew H. Sung
}

\begin{abstract}
Smartphones are widely used today, and it becomes possible to detect the user's environmental changes by using the smartphone sensors, as demonstrated in this paper where we propose a method to identify human activities with reasonably high accuracy by using smartphone sensor data. First, the raw smartphone sensor data are collected from two categories of human activity: motion-based, e.g., walking and running; and phone movement-based, e.g., left-right, up-down, clockwise and counterclockwise movement. Firstly, two types of features extraction are designed from the raw sensor data, and activity recognition is analyzed using machine learning classification models based on these features. Secondly, the activity recognition performance is analyzed through the Convolutional Neural Network (CNN) model using only the raw data. Our experiments show substantial improvement in the result with the addition of features and the use of CNN model based on smartphone sensor data with judicious learning techniques and good feature designs.
\end{abstract}

Index Terms-CNN, feature extraction, human activity recognition, sensors, smartphone, SVM.

\section{INTRODUCTION}

Nowadays mobile devices have become a most important tool in people's daily life for communication. Older generation mobile phones did not offer much capability to the user more than passive communications; smartphones then came into play and increasingly provided intelligent assistance to the user in their everyday activities. In addition to the mobile operating system with computing ability and interconnectivity, application programming interfaces(API) for running third party tools and applications, smartphones have features such as cameras, GPS, web browsers, etc., and the embedded sensors such as accelerometers, gyroscope and magnetometer [1] which allow the development of apps based on the user's context, location, motion, etc.

To develop intelligent smartphone apps, it is important to utilize context recognition and situational awareness of the device's user. Activity recognition is one such platform for these devices which can be handled by the built-in sensors

Manuscript received January 22, 2018; revised April 5, 2018.

Q. Liu and P. Uduthalapally are with the Department of Computer Science, Sam Houston State University, Huntsville, TX 77382 USA.0523 USA (e-mail: pxu001@shsu.edu, liu@shsu.edu).

Z. Zhou, S. R. Shakya, and A. H. Sung are with the School of Computing, University of Southern Mississippi, Hattiesburg, MS 39406 USA (e-mail: sarbagya.shakya@usm.edu,zhaoxian.zhou@usm.edu,Andrew.sung@usm.e $\mathrm{du})$.

M. Qiao is with the Mathematics and Computer Science, South Dakota School of Mines and Technology, Rapid City, SD 57701 USA (e-mail: mengyu.qiao@sdsmt.edu). and are already used in different sectors like business, healthcare, safety, transportation, etc. Other types of sensors include wearable sensors which detect the movement, and the Bluetooth sensor which enables the transfer of data from one device to another using the data communication channels [2]-[4]. Sensing and recording become possible with these mobile sensors which help to monitor the subjects constantly. Activities can also be stored when monitored in their preferred environment [5].

The data collected from sensors can be used to determine the state and activity of a person independent of external infrastructure. This is referred to human activity recognition and has drawn importance in every walk of life with increase of its usage in applications such as monitoring the health care and security of the patients or elderly people who live alone. It helps to assist them in need, to improve the quality of life, freedom and security. There has been a rapid advancement in this research field, one of the applications that received attention is introducing the human activity recognition benefit to athletes and health concerned patients. Here it helps to gather information of the athlete's activities, identify the injuries and report to the family or associated doctor for treatment.

Most of the research is focused on the motion-related activities like standing, running, walking and other activities like storing data based on the phone movements performed by a set of users within a particular time frame. Here in this research, accelerometers are used as major part of the human activity recognition which helps to identify walking, running motions of the users easily. The famous and interesting research that has been carried out by using smartphone sensors are monitoring the patient's behaviors and current mobility.

Many smartphones based activity recognition has demonstrated the importance of research in the field. There are many similar approaches carried out but our approach, collection and evaluation of most of the embedded sensors based on the activities has not been done previously and is advantageous than other approaches. A most widespread problem of context recognition in mobile device is the orientation of the device, i.e., how the device is carried? As it greatly affects the analysis that performed to recognize the activities and will lead to inaccurate data.

The rest of the paper is summarized as follows: Section II gives an overview of the literature review --- the past and the recent development in this field; Section III describes the proposed methodology and Section IV describes our feature design. Section V deals with the experiment results and Section VI illustrates the conclusion drawn from the experiments, giving light for future study. 


\section{RELATED WORK}

There has been a rapid increase in the number of mobile apps in recent years due to the popularity of smartphones and the app-stores. But there has been only limited work on the apps dealing with the sensing devices due to the constraints imposes on implementing communications between the smartphone and the sensors. It has also been a challenging issue to handle the external sensors communication as it leads to energy and deployment issues. It led to focus on the two important areas of research: 1. control the programming barriers, and 2. make the mobile sensing applications efficient and simpler to deploy. This paper is focused on the device sensors to provide better customization and flexibility that apps can utilize.

Activity recognition is not a new topic. Bao and Intille [6] developed a system which is used to identify the activities performed using bi-axial accelerometers when placed at five locations on the user's body. There were similar works carried out by [2], [7]-[10] to identify the user activities with the use of accelerometer. Other works focused on the applications built on the activity recognition which helps to promote health and fitness[6]. Krishnan et al. [11] conducted experiments by collecting data from three users using two accelerometers for determining five activities; the paper concluded that there is a need to use two accelerometers as the thigh accelerometer data is not sufficient for recognizing activities like sitting, running, lying down etc. Later in another paper Krishnan [12] conducted experiments by using three wearable accelerometers to examine seven lower body activities from data collected from ten subjects. Mannini \& Sabitini [13] experimented by using five tri-axial accelerometers and learning methods were used to recognize the postures and movements of the users. Hoseini-tabatabaei et al. [14] based on the research work on the human activity recognition has summarized the related research data and the approaches used in context awareness in the smartphones. As the research work continued, suggestions were made to perform the similarity search between the patterns to identify the activities.

Later, researchers have used combination of accelerometer and other sensors for activity recognition. Parker introduced a system to recognize the activities (standing, walking, running, playing ball, using the toilet at particular locations) performed. User's location and activities recognition were studied by Lee \& Mase [15]. Studies also focused on using other available sensors in addition to accelerometers. A "ewatch" device is placed in shirt pocket, trouser pocket, backpack etc. to recognize the common user activities, with the use of bi-axial accelerometer and light sensor by Maurer et al.[16]. Choudhury et al. [8] used a model consisting of seven different sensors to recognize activities. Liu et al.[17] have adopted smartphone sensors to analyze vehicle and pedestrian behaviors.

Recently there have been studies focused on the usage of single or multiple accelerometers because most of the common activities, walking, running, jogging etc., can be recognized easily with them. Machine learning techniques such as k-Nearest Neighbor, Naive Bayes, Decision Trees, Support Vector Machines(SVM), Neural Networks, etc. can be used for learning and feature extraction for identifying the activities. Artificial intelligence, machine learning and recently deep learning approaches have been used in studies showing effective results in identifying different activities from body-fixed sensor data [4], [9], [18]-[20]. A wide variety of approaches has been used to extract the features form the accelerometer data directly using time-varying acceleration signal[4] and frequency analysis [15], [21], [22]. Wavelet analysis is also used to derive the features[18]. Recently Mohammad Abu Alsheikh[23] have analyzed the activity recognition in some datasets and have implemented deep learning paradigms and have shown recognition improvement than other state-of-the art methods in human activity recognition. Gjoreski et al. [24] have analyzed the classical machine learning and deep learning methods and have shown that deep learning algorithm have slightly outperformed the machine learning methods. Also Baccouche [25] have proposed a fully automated deep model without any prior knowledge for an video based dataset. The recent advancement of deep learning have also made it possible to produce promising results in different areas with high level features extraction [26].

In this paper, the main work is focused on the study of activity recognition based on smartphones sensors. The concept of similarity measure is applied to detect and predict the activities to improve the recognition accuracy.

\section{Methodology}

This section presents our proposed methods for activity recognition. The methodology can be divided into two phases. Phase 1 consists of collecting the raw sensor data from the accelerometer, which detects the motions and the direction of the mobile phone pointing to, and the gyroscope that senses rotational motions and orientation, which are generally difficult to sense by humans. In phase 2 , we first describe the feature extraction method performed to the raw data and machine learning models built for activity detection, then we describe our deep learning model based on convolutional neural network.

\section{A. Machine Learning Model}

The main steps in this section include:

1) Sensor data filtering - outputs the filtered sensor data based on individual activities performed by the user.

2) Sensor data segmentation — gives the filtered data that divide the data into segments that are related to the activity.

3) Feature extraction - we designed two types of features that are used for classification.

4) Classification - machine learning techniques, SVM classifier with linear kernel (LibSVM) and Fisher linear discriminants (FLD) are used to construct supervised classification model.

5) Detection/Prediction — the human activities are predicted based on the features extracted and established classification models.

In this process, the data collected from the accelerometer and the gyroscope, are firstly preprocessed with noise filters and then sampled with a fixed size window of 2.5 seconds to extract the features. The time window size is determined empirically to achieve the detection in real -time. If the window size is too small, there is no enough data to profile 
robust features and if the window size is too large, the robustness is enhanced however the decision is accordingly delayed, not in a real- time manner. The data is collected from different age-group users and $60 \%$ of collected data is used as training set and other $40 \%$ as testing set. The detection error percentages are recorded by using LibSVM and FLD.

\section{B. Deep Learning Models}

In this section, the time sequence raw data collected from the accelerometer and gyroscope of the mobile device are passed through the deep activity (CNN) model after normalization.

The time series raw data is divided into many fixed sized segments with each component along the third dimension. The window of size 100 is used which is moved over the raw data in a fixed step with $50 \%$ overlapping data. The input dimension of the data in our model is $[317,100,6]$ where 317 is the number of segments. 100 is the window size and 6 is the number of channels. Then we reshaped our input data accordingly to feed into our model. The class label having the maximum number of count in the window is selected as the label for that segment. The dataset is divided into $80 \%$ of training dataset and $20 \%$ of testing dataset.

The model consists of two convolution layers, pooling layer, fully connected layer, dropout layer and SoftMax layer. In convolution layers, depth wise convolution was applied with several convolution filters. Then ReLU activation function is applied to the output of the convolution layer to introduce nonlinearity to a system. In pooling layer max pooling algorithm is used to reduce the spatial size with filter of size $2 \times 2$. Then a fully connected layer is applied followed by dropout layer to avoid overfitting of training data. SoftMax regression was performed in the SoftMax layer at the end. The optimizer used in this model is Adam optimizer.

\section{FEATURE EXTRACTION}

Feature design plays very important role in machine learning and pattern recognition applications. In this study, our feature design is described below.

Let $f(t)$ denote the sensor data signal over the time t and $s(t)$ denote the first derivative of $\mathrm{f}(\mathrm{t})$, that is, $s(t)=f(t+1)-f(t)$, where $t=(0,1,2, \cdots, T-1)$. We have designed two feature extraction method. The first type features are denoted as sf, containing the following features.

1. The minimal values of $f(t)$ and $s(t)$;

$$
\begin{aligned}
& s f_{f}(1)=\min (f) \\
& s f_{s}(1)=\min (s)
\end{aligned}
$$

2. The maximal values of $f(t)$ and $s(t)$;

$$
\begin{aligned}
& s f_{f}(2)=\max (f) \\
& s f_{s}(2)=\max (s)
\end{aligned}
$$

3. The mean values of $|f(t)|$ and $|s(t)|$;

$$
\begin{aligned}
& s f_{f}(3)=\operatorname{mean}(|f|) \\
& s f_{s}(3)=\operatorname{mean}(|s|)
\end{aligned}
$$

4. The variance values of $f(t)$ and $s(t)$;

$$
s f_{f}(4)=\operatorname{var}(f)
$$

$$
s f_{s}(4)=\operatorname{var}(s)
$$

5. The probability of $f(t)$ and $s(t)$ with the values in the segmentation zones that are equally divided $f(t)$ and $s(t)$. We assume the range of $f(t) \mathrm{RF}$ and the range of $s(t) \mathrm{RS}$, the number of segmentation zones is $N$, the occurrence of $f(t)$ with the values in the zone $i$ (the range $[\min (f)+(i-1) *$ $\mathrm{RF} / \mathrm{N}, \min (f)+i * \mathrm{RF} / \mathrm{N}]$, where $i=1,2, \cdots, N)$ is $c f(i)$, and the occurrence of $s(t)$ with the values in the zone $i$ (the $\operatorname{range}[\min (s)+(i-1) * \mathrm{RS} / \mathrm{N}, \min (\mathrm{s})+i * \mathrm{RS} / \mathrm{N}])$ is $c s(i)$, then,

$$
\begin{aligned}
& s f_{f}(4+i)=\frac{c f(i)}{\sum_{i} c s(i)} \\
& s f_{s}(4+i)=\frac{c s(i)}{\sum_{i} c s(i)}
\end{aligned}
$$

In our experiments, $\mathrm{N}$ is set to 20 . We have a total of 48 features in the sf feature set.

Our second type features are based on the Fourier transform of the sensor signals, denoted as fc in our study.

Let $F(x)$ and $S(x)$ denote the magnitude of the Fourier transform of $f(t)$ and $s(t)$, the Fourier domain-based features $H C F_{f}$ and $H C F_{s}$ are calculated by the following algorithm:

$$
H C F_{f}=0 ; \quad H C F s=0 \text {; }
$$

For $m=1$ : round(length $(F(x)) / 2)$

$H C F_{f}=H C F_{f}+F(m) * m$

end

For $m=1$ : round(length $(S(x)) / 2)$

$H C F s=H C F s+S(m) * m$

End

\section{EXPERIMENTS}

For the experimental analysis, we collected the sensor data for the following human activities: running, walking, clockwise movement (CW), counter clockwise movement (CCW), up \& down (UD), and left \& right (LR). The raw data collected consist of raw time series data with $\mathrm{X}, \mathrm{Y}, \mathrm{Z}$ axis values for each label. Each category of human activity contains 50 event samples. The experiments were carried out by placing the phone in the pants pocket, holding it in hand and by placing it on ground. The data collected were carefully observed and later the data were sampled at a frequency of 25 $\mathrm{Hz}$.

\section{A. Machine Learning Classifiers Results}

Tables I and II list the mean detection accuracy by using LibSVM and FLD respectively. Detection accuracy here is given as 1 minus the error value based on the accelerometer and gyroscope sensors, respectively, by distinguishing one event from another over 30 experiments for each binary classification. In this experiment, the $60 \%$ training set is randomly selected. The results from the experiment show that the mean detection accuracy can be found maximum of $100 \%$ for different set of events. Among different set of features used, A/G-sf shows the maximum number of highest mean detection accuracy (10 among 15 set of events) using lib SVM and A-fc (6 among 15 set of events) by using FLD. The other highest accuracy for each set of events also have that features using accelerometer data or both accelerometer and gyroscope data. Table III lists the mean values of confusion matrix over 30 experiments by applying LibSVM to AG-sf features.

The experimental results show that the statistical features 
generally lead to higher detection accuracy. LibSVM performs better in almost all set of events than FLD in terms of the classification accuracy. The accelerometer sensor features are better and contribute more towards the accuracy of the results than gyroscope sensor features in most detections. Also, when combine data are used, the detection accuracy can also improve.

TABLE I: MEAN DeTECTION ACCURACY \pm THE STANDARD DEVIATION (\%) OVER 30 EXPERIMENTS BY USING LIBSVM

\begin{tabular}{|c|c|c|c|c|c|c|c|c|c|c|}
\hline \multicolumn{2}{|c|}{ Activity Recognition } & \multirow[b]{2}{*}{ A-sf } & \multirow[b]{2}{*}{ G-sf } & \multirow[b]{2}{*}{ A/G-sf } & \multirow[b]{2}{*}{ A-fc } & \multirow[b]{2}{*}{ G-fc } & \multirow[b]{2}{*}{ A/G-fc } & \multirow[b]{2}{*}{ A-sf/fc } & \multirow[b]{2}{*}{ G-sf/fc } & \multirow{2}{*}{$\begin{array}{l}\text { A/G- } \\
\text { sf/fc }\end{array}$} \\
\hline Event A & Event B & & & & & & & & & \\
\hline Run & Walk & $100 \pm 0$ & $93.2 \pm 6.6$ & $100 \pm 0$ & $96.2 \pm 3.8$ & $83.8 \pm 7.3$ & $97.9 \pm 3.5$ & $96.8 \pm 3.4$ & $83.5 \pm 8.6$ & $97.9 \pm 3.5$ \\
\hline Run & Left right & $99.9 \pm 0.5$ & $99.7 \pm 0.8$ & $100 \pm 0$ & $95.4 \pm 2.8$ & $93.4 \pm 3.7$ & $99.5 \pm 2.1$ & $95.4 \pm 3.1$ & $94.0 \pm 3.7$ & $99.5 \pm 1.3$ \\
\hline Run & Up down & $100 \pm 0$ & $90.7 \pm 7.4$ & $100 \pm 0$ & $94.9 \pm 3.0$ & $73.1 \pm 6.0$ & $94.8 \pm 3.1$ & $93.2 \pm 4.5$ & $76.6 \pm 10.1$ & $96.0 \pm 2.3$ \\
\hline Run & Clockwise & $96.3 \pm 2.2$ & $85.3 \pm 7.4$ & $96.1 \pm 2.5$ & $96.7 \pm 3.0$ & $72.1 \pm 4.0$ & $97.3 \pm 2.6$ & $97.2 \pm 1.9$ & $80.0 \pm 8.6$ & $97.7 \pm 2.5$ \\
\hline Run & Counterclockwise & $96.3 \pm 2.6$ & $93.7 \pm 3.8$ & $96.2 \pm 1.9$ & $96.3 \pm 2.8$ & $78.9 \pm 8.8$ & $96.9 \pm 3.0$ & $96.2 \pm 2.8$ & $77.8 \pm 8.6$ & $97.2 \pm 2.4$ \\
\hline Walk & Left right & $84.0 \pm 3.7$ & $97.8 \pm 2.0$ & $97.3 \pm 2.7$ & $71.4 \pm 2.2$ & $75.1 \pm 13.1$ & $85.9 \pm 5.5$ & $78.5 \pm 6.8$ & $81.0 \pm 9.1$ & $87.4 \pm 6.0$ \\
\hline Walk & Up down & $99.4 \pm 1.2$ & $94.8 \pm 3.6$ & $99.0 \pm 1.4$ & $97.9 \pm 2.4$ & $89.0 \pm 8.7$ & $96.6 \pm 2.6$ & $97.7 \pm 2.7$ & $91.6 \pm 6.8$ & $97.4 \pm 2.7$ \\
\hline Walk & Clockwise & $98.0 \pm 2.3$ & $96.1 \pm 3.9$ & $97.5 \pm 2.9$ & $75.0 \pm 1.1$ & $81.1 \pm 7.2$ & $85.6 \pm 5.4$ & $93.5 \pm 4.8$ & $82.3 \pm 6.6$ & $87.8 \pm 5.4$ \\
\hline Walk & Counterclockwise & $98.1 \pm 2.6$ & $96.2 \pm 2.9$ & $98.1 \pm 2.6$ & $79.6 \pm 6.6$ & $86.4 \pm 8.7$ & $89.6 \pm 4.6$ & $92.3 \pm 4.8$ & $88.5 \pm 8.5$ & $89.9 \pm 5.2$ \\
\hline Left right & Up down & $99.2 \pm 1.0$ & $98.1 \pm 1.5$ & $99.4 \pm 0.9$ & $99.3 \pm 1.3$ & $59.7 \pm 20.8$ & $97.9 \pm 1.9$ & $98.8 \pm 1.2$ & $88.6 \pm 16.2$ & $98.0 \pm 1.9$ \\
\hline Left right & Clockwise & $95.5 \pm 2.6$ & $98.2 \pm 1.3$ & $98.6 \pm 1.0$ & $77.8 \pm 7.7$ & $66.3 \pm 17.1$ & $86.1 \pm 4.9$ & $94.4 \pm 4.2$ & $93.7 \pm 8.8$ & $89.4 \pm 4.8$ \\
\hline Left right & Counterclockwise & $97.4 \pm 1.6$ & $100 \pm 0$ & $100 \pm 0$ & $89.9 \pm 14.1$ & $90.1 \pm 8.4$ & $97.2 \pm 2.1$ & $94.8 \pm 5.1$ & $94.4 \pm 2.9$ & $97.7 \pm 1.8$ \\
\hline Up down & Clockwise & $99.2 \pm 0.9$ & $75.7 \pm 14.0$ & $99.4 \pm 0.9$ & $99.1 \pm 1.3$ & $52.7 \pm 4.4$ & $98.3 \pm 1.4$ & $98.9 \pm 1.4$ & $65.8 \pm 14.7$ & $98.5 \pm 1.5$ \\
\hline Up down & Counterclockwise & $98.9 \pm 1.0$ & $63.1 \pm 13.7$ & $98.9 \pm 1.0$ & $98.8 \pm 1.2$ & $47.6 \pm 7.8$ & $98.3 \pm 1.6$ & $98.6 \pm 1.5$ & $48.7 \pm 19.3$ & $98.6 \pm 1.7$ \\
\hline Clockwise & Counterclockwise & $85.9 \pm 3.7$ & $55.3 \pm 4.1$ & $85.7 \pm 3.8$ & $55.4 \pm 4.9$ & $52.1 \pm 3.0$ & $54.9 \pm 4.7$ & $83.8 \pm 4.2$ & $55.1 \pm 6.0$ & $83.0 \pm 4.1$ \\
\hline
\end{tabular}

TABLE II: MEAN DETECTION ACCURACY \pm THE STANDARD DEVIATION (\%) OVER 30 EXPERIMENTS BY USING FLD

\begin{tabular}{|c|c|c|c|c|c|c|c|c|c|c|}
\hline \multicolumn{2}{|c|}{ Activity Recognition } & \multirow[b]{2}{*}{ A-sf } & \multirow[b]{2}{*}{ G-sf } & \multirow[b]{2}{*}{ A/G-sf } & \multirow[b]{2}{*}{ A-fc } & \multirow[b]{2}{*}{ G-fc } & \multirow[b]{2}{*}{ A/G-fc } & \multirow[b]{2}{*}{ A-sf/fc } & \multirow[b]{2}{*}{ G-sf/fc } & \multirow{2}{*}{$\begin{array}{l}\text { A/G- } \\
\text { sf/fc }\end{array}$} \\
\hline Event A & Event B & & & & & & & & & \\
\hline Run & Walk & $91.6 \pm 6.5$ & $91.9 \pm 6.0$ & $95.1 \pm 3.9$ & $96.4 \pm 3.7$ & $88.1 \pm 6.4$ & $96.0 \pm 4.5$ & $92.7 \pm 5.3$ & $92.2 \pm 5.1$ & $95.7 \pm 3.8$ \\
\hline Run & Left right & $97.3 \pm 2.8$ & $89.3 \pm 5.3$ & $95.5 \pm 3.8$ & $96.8 \pm 2.7$ & $94.5 \pm 3.1$ & $98.1 \pm 3.1$ & $97.2 \pm 3.0$ & $93.2 \pm 4.7$ & $94.3 \pm 5.6$ \\
\hline Run & Up down & $98.7 \pm 2.1$ & $96.4 \pm 4.2$ & $97.3 \pm 2.7$ & $95.3 \pm 3.5$ & $85.4 \pm 5.8$ & $97.3 \pm 2.7$ & $98.6 \pm 2.3$ & $95.3 \pm 3.8$ & $96.9 \pm 2.8$ \\
\hline Run & Clockwise & $93.5 \pm 3.5$ & $85.9 \pm 6.3$ & $94.9 \pm 4.3$ & $98.1 \pm 2.2$ & $78.1 \pm 4.7$ & $97.9 \pm 3.0$ & $95.8 \pm 4.0$ & $89.0 \pm 5.5$ & $94.0 \pm 4.6$ \\
\hline Run & Counterclockwise & $89.4 \pm 6.1$ & $90.7 \pm 4.2$ & $93.5 \pm 4.3$ & $97.1 \pm 2.7$ & $89.1 \pm 4.1$ & $96.3 \pm 2.5$ & $90.8 \pm 6.8$ & $89.8 \pm 6.2$ & $92.3 \pm 4.3$ \\
\hline Walk & Left right & $67.2 \pm 8.2$ & $84.0 \pm 4.1$ & $85.8 \pm 6.5$ & $71.2 \pm 5.9$ & $88.1 \pm 4.8$ & $89.3 \pm 5.5$ & $67.6 \pm 8.6$ & $82.5 \pm 6.4$ & $87.2 \pm 6.3$ \\
\hline Walk & Up down & $98.4 \pm 2.0$ & $95.3 \pm 3.7$ & $94.6 \pm 3.1$ & $97.4 \pm 3.3$ & $86.3 \pm 6.7$ & $96.6 \pm 3.0$ & $97.2 \pm 2.9$ & $93.8 \pm 3.1$ & $95.0 \pm 3.2$ \\
\hline Walk & Clockwise & $93.2 \pm 5.3$ & $89.7 \pm 4.5$ & $93.3 \pm 5.1$ & $81.7 \pm 4.8$ & $81.3 \pm 4.9$ & $81.5 \pm 4.9$ & $92.0 \pm 4.2$ & $90.6 \pm 5.0$ & $90.9 \pm 5.1$ \\
\hline Walk & Counterclockwise & $87.0 \pm 5.1$ & $94.9 \pm 4.3$ & $94.7 \pm 4.0$ & $87.8 \pm 4.1$ & $90.5 \pm 4.6$ & $90.1 \pm 3.7$ & $88.6 \pm 5.5$ & $94.8 \pm 3.8$ & $93.4 \pm 4.1$ \\
\hline Left right & Up down & $94.1 \pm 4.3$ & $94.2 \pm 3.5$ & $98.2 \pm 1.9$ & $99.3 \pm 0.9$ & $88.4 \pm 3.3$ & $97.9 \pm 1.8$ & $96.7 \pm 2.5$ & $95.0 \pm 3.3$ & $95.6 \pm 2.8$ \\
\hline Left right & Clockwise & $88.2 \pm 4.5$ & $94.0 \pm 3.5$ & $97.7 \pm 2.4$ & $85.5 \pm 4.1$ & $87.0 \pm 3.7$ & $91.7 \pm 3.4$ & $88.9 \pm 4.6$ & $94.1 \pm 4.0$ & $94.6 \pm 4.1$ \\
\hline Left right & Counterclockwise & $85.7 \pm 5.5$ & $95.8 \pm 2.8$ & $96.5 \pm 3.2$ & $91.6 \pm 3.4$ & $92.7 \pm 2.8$ & $96.7 \pm 2.4$ & $85.1 \pm 6.5$ & $95.9 \pm 3.5$ & $95.6 \pm 2.6$ \\
\hline Up down & Clockwise & $94.7 \pm 3.8$ & $81.7 \pm 4.8$ & $92.9 \pm 4.2$ & $99.0 \pm 1.1$ & $62.7 \pm 6.7$ & $98.7 \pm 1.5$ & $94.9 \pm 3.1$ & $84.5 \pm 5.1$ & $94.7 \pm 2.9$ \\
\hline Up down & Counterclockwise & $96.1 \pm 3.2$ & $75.6 \pm 5.8$ & $94.3 \pm 4.2$ & $98.7 \pm 1.4$ & $53.9 \pm 8.0$ & $98.1 \pm 2.2$ & $96.7 \pm 2.2$ & $75.6 \pm 6.0$ & $96.1 \pm 3.2$ \\
\hline Clockwise & Counterclockwise & $72.2 \pm 7.3$ & $72.9 \pm 7.0$ & $73.5 \pm 5.5$ & $62.6 \pm 5.3$ & $58.2 \pm 6.9$ & $62.2 \pm 6.1$ & $72.1 \pm 7.3$ & $69.9 \pm 6.4$ & $74.4 \pm 5.6$ \\
\hline
\end{tabular}

TABLE III: Multiple ClassificATION CONFusion MATRIX By USING A/G-SF FEATURES with LIBSVM

\begin{tabular}{c|cccccc}
\hline & Run & walk & LR & UD & CW & 0.0030 \\
\hline Run & 0.9 & 0 & 0 & 0 & 0.097 \\
\hline Walk & 0 & 0.9 & 0.0400 & 0 & 0.0067 \\
\hline LR & 0 & 0.0103 & 0.9756 & 0 & 0.0141 \\
\hline UD & 0 & 0 & 0.0026 & 0.9782 & 0.0192 & 0.8644 \\
\hline CW & 0.0078 & 0 & 0.0244 & 0.0011 & 0 \\
\hline
\end{tabular}

\section{B. Deep Learning Models}

For deep learning models, we performed the analysis for the data consisting of the time sequence accelerometer and gyroscope values taken from the sensors of the mobile device.

Fig. 1 shows the output graph for the accuracy and loss curve for the deep learning CNN model. The curve shows that the accuracy increases and remains constant to the final value of $98.1 \%$ at the end of the iteration. Also, the loss curve shows that the loss has continuously decreased throughout the iteration process.

In the study, we experimented the model by using only the accelerometer data and only the gyroscope data, respectively. The prediction accuracy for the accelerometer data only was found to be $82.8 \%$ and with gyroscope only was found to be $76 \%$. It is concluded that the activity recognition accuracy can be enhanced by using the deep learning model using data from both accelerometer and gyroscope.

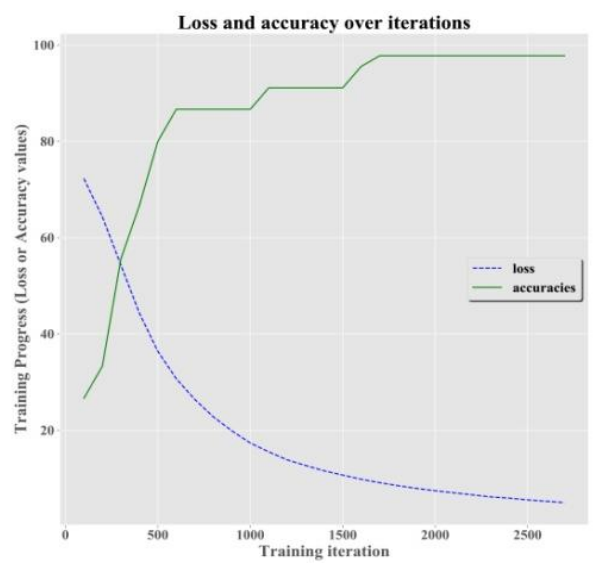

Fig. 1. Loss accuracy curve. 


\section{CONCLUSION}

In this research, we designed an experiment of for human activity recognition based on data from smart phone sensors. We implemented a dataset, and designed two types of features to recognize human activity and smartphone motion based on the accelerometer and gyroscope sensors data. Our study shows that the proposed gestures are effective for the intended detection. Binary classification of the events was performed to produce better and effective results when compared to performing individual events as it results in negligible amount of inaccuracy. Combination of events resulted in higher error rate when detection accuracy is analyzed. From the results, it can be concluded that accelerometer sensor(A-sensor) reading contribute more compared to G-sensor reading (Gyroscope sensor) but can increase the accuracy of the detection when used both. We analyzed our result by using both machine learning and deep learning algorithms.

The results also proved to show consistency on the mobile device uncommon orientations. Phone movements are calculated with the use of both the internal sensors. It can be expected that the scope of the activities recognition can be expanded with the use of more collected data with multi class and multi label datasets and can be implemented to make it more useful for app development to benefit users.

\section{REFERENCES}

[1] N. D. Lane, E. Miluzzo, H. Lu, D. Peebles, T. Choudhury, and A. T. Campbell, "A survey of mobile phone sensing," IEEE Commun. Mag., vol. 48, pp. 140-150, Sep. 2010.

[2] T. Brezmes, J. L. Gorricho, and J. Cotrina, "Activity recognition from accelerometer data on mobile phones," in IWANN '09: Proc. the $10^{\text {th }}$ International Work Conference on Artificial Neural Networks, 2009, pp. 796-799.

[3] N. Gyorbiro, A. Fabian, and G. Homanyi, "An activity recognition system for mobile phones," in MONET, 2009.

[4] T. S. Saponas, J. Lester, J. E. Froehlich et al., "Ilearn on the Iphone: Real-time human activity classification on commodity mobile phones," CSE Technical Report, 2008.

[5] J. Goldman et al., "Participatory sensing: A citizen-powered approach to illuminating the patterns that shape our world," 2009.

[6] L. Bao and S. S. Intille, "Activity recognition from user-annotated acceleration data," in Proc. Pervasive 2004, 2004, pp. 1-17.

[7] Y. Cho, Y. Nam, Y.-J. Choi, and W.-D. Cho, "SmartBuckle: Human activity recognition using a 3-axis accelerometer and a wearable camera," HealthNet, 2008.

[8] T. Choudhury et al., "The mobile sensing platform: An embedded activity recognition system," IEEE Pervasive Comput., vol. 7, pp. $32-41,2008$

[9] N. Gyorbiro, A. Fabian, and G. Homanyi, "An activity recognition system for mobile phones," Mob. Networks Appl., vol. 14, pp. 82-91, 2008.

[10] A. Anjum and M. U. Ilyas, "Activity recognition using smartphone sensors," in Proc. the IEEE $10^{\text {th }}$ Consumer Communications and Networking Conference (CCNC '13), 2013, pp. 914-919.

[11] N. Krishnan, D. Colbry, C. Juillard, and S. Panchanathan, "Real time human activity recognition using tri-Axial accelerometers," in Proc. Sensors, Signals and Information Processing Workshop, 2008.

[12] N. Krishnan and S. Panchanathan, "Analysis of low resolution accelerometer data for continuous human activity recognition," in Proc. IEEE International Conference on Acoustics, Speech and Signal Processing, 2008, pp. 3337-3340.

[13] A. Mannini and A. M. Sabatini, "Machine learning methods for classifying human activity from onbody accelerometers," Sensors, vol. 10, pp. 1154-1175, 2010

[14] S. A. Hoseini-Tabatabaei, A. Gluhak, and R. Tafazolli, "A Survey on smartphone-based systems for opportunistic user context recognition," ACM Comput. Surv., vol. 45, p. 27, 2013.

[15] S.-W. Lee and K. Mase, "Activity and location recognition using wearable sensors," IEEE Pervasive Comput., vol. 1, pp. 24-32, 2002.
[16] U. Maurer, A. Smailagic, D. P. Siewiorek, and M. Deisher, "Activity recognition and monitoring using multiple sensors on different body positions," in Proc. International Workshop on Wearable and Implantable Body Sensor Networks (BSN'06), 2006, p. 4, p. 116.

[17] S. Bhattacharya and N. D. Lane, "From smart to deep: Robust activity recognition on smartwatches using deep learning," in Proc. 2016 IEEE Int. Conf. Pervasive Comput. Commun. Work, 2016, pp. 1-6.

[18] I. Anderson et al., "Shakra: Tracking and sharing daily activity levels with unaugmented mobile phones," Mob. Networks Appl., vol. 12, pp. 185-199, Aug. 2007.

[19] C. A. Ronao and S.-B. Cho, "Deep convolutional neural networks for human activity recognition with smartphone sensors," Neural Information Processing, pp. 46-53, 2015.

[20] N. Y. Hammerla, S. Halloran, and T. Plotz, "Deep, convolutional, and recurrent models for human activity recognition using wearables," in Proc. the $25^{\text {th }}$ International Conference on Artificial Intelligence, 2016, pp. 1533-1540.

[21] J. R. Kwapisz, G. M. Weiss, and S. A. Moore, "Activity recognition using cell phone accelerometers," ACM SIGKDD Explor. Newsl., vol. 12, pp. 74-82, 2011.

[22] M. Berchtold, M. Budde, D. Gordon, H. R. Schmidtke, and M. Beigl, "ActiServ: Activity recognition service for mobile phones," in Proc. International Symposium on Wearable Computers, 2010, pp. 1-8.

[23] M. A. Alsheikh, A. Selim, D. Niyato, L. Doyle, S. Lin, and H.-P. Tan, "Deep activity recognition models with triaxial accelerometers," ArXiv e-prints, Nov. 2015.

[24] H. Gjoreski, J. Bizjak, M. Gjoreski, and M. Gams, "Comparing deep a classical machine learning methods for human activity recognition using wrist accelerometer," in Proc. the $25^{\text {th }}$ International Conference on Artificial Intelligence, 2016, pp. 1-7.

[25] M. Baccouche, F. Mamalet, C. Wolf, C. Garcia, and A. Baskurt, "Sequential deep learning for human action recognition," in Proc. the Second International Conference on Human Behavior Unterstanding, 2011, pp. 29-39.

[26] J. Wang, Y. Chen, S. Hao, X. Peng, and L. Hu, "Deep learning for sensor-based activity recognition: A survey," CoRR, vol. abs/1707.0, 2017.

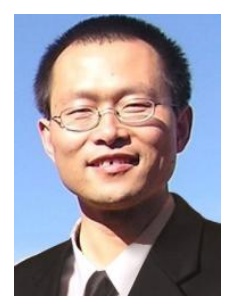

Qingzhong Liu is currently an associate professor in Computer Science at Sam Houston State University (SHSU). He earned his $\mathrm{PhD}$ degree in computer science from New Mexico Institute of Mining and Technology in 2007. His research interests include information assurance, digital forensics, multimedia computing and analysis, bioinformatics, pattern recognition, machine intelligence and applications. His study in multimedia forensics has been funded by the National Institute of Justice and the National Science Foundation. He is the recipient of the Greater Houston Fraud Impact Award in 2015, and the SHSU Faculty Excellence Award in Scholarly and Creative Accomplishments in 2017. He has been serving on the technical program committee for ACM Workshop on Information Hiding and Multimedia Security since 2013.

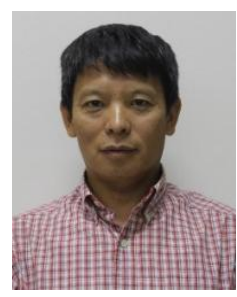

Zhaoxian Zhou received the B. Eng. from the University of Science and Technology of China in 1991; M. Eng. from the National University of Singapore in 1999 and the $\mathrm{PhD}$ degree from the University of New Mexico in 2005. All degrees are in electrical engineering. From 1991 to 1997, he was an electrical engineer in China Research Institute of Radiowave Propagation. He joined the University of Southern Mississippi in 2005. He has published more than forty papers in academic journals conference proceedings. His current research interests include computational science and electrical engineering.

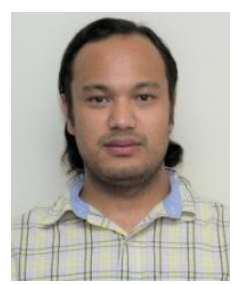

Sarbagya Ratna Shakya received the B. Eng. in electronics engineering from National College of Engineering, Tribhuvan University of Nepal in 2009; M. Eng. in computer engineering from Nepal College of Information Technology, Pokhara University of Nepal in 2014. Currently he is a PhD student in School of Computing, University of Southern Mississippi (USM) since 2015. His current research interest includes machine learning, deep learning, high performance computing. 


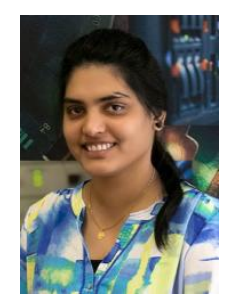

Prathyusha Uduthalapally obtained her master degree in computer science from Sam Houston State University (SHSU) in 2016. She is currently a database administrator at SHSU.

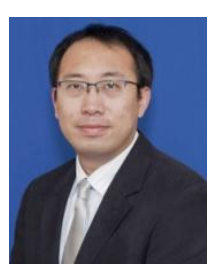

Mengyu Qiao received the M.S. degree in 2009 and the $\mathrm{Ph} . \mathrm{D}$. degree in 2011 in computer science from New Mexico Institute of Mining and Technology. He joined the Mathematics and Computer Science Department of South Dakota School of Mines and Technology in 2011 as an assistant professor and was promoted to Associate Professor in 2017. He is also a senior researcher at the Center for Security Printing and Anti-Counterfeiting Technology of SDSMT. His research interests include, but not limited to, cybersecurity, multimedia processing, digital forensics, mobile computing, bioinformatics, machine intelligence and computational applications.

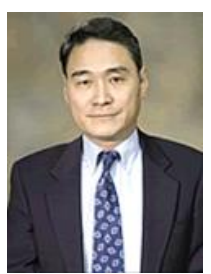

Andrew H. Sung received B.S. degree in electrical engineering from National Taiwan University, M.S. degree in mathematical sciences from University of Texas at Dallas, and Ph.D. degree in computer science from State University of New York at Stony Brook. He has served in the Air Force of Taiwan as second lieutenant for 2 years, and assistant, associate, and professor of Computer Science and Engineering in three public universities in the U.S. over 30 years. Since 2014, he has been the director and professor of the School of Computing, The University of Southern Mississippi, in Hattiesburg, Mississippi. Dr. Sung has over 200 publications and is currently working in the areas of data mining, multimedia forensics, and cybersecurity. 